Artículo original

\title{
Estructura de la población del cangrejo rey Damithrax spinosissimus en el Caribe colombiano
}

\author{
Néstor Hernando Campos¹, Ana Milena Lagos¹, Adriana Bermúdez², Edna Márquez ${ }^{3}$ \\ ${ }^{1}$ CECIMAR, Universidad Nacional de Colombia, Sede Caribe, Santa Marta, Colombia \\ ${ }^{2}$ Universidad de Cartagena \\ ${ }^{3}$ Universidad Nacional de Colombia, Sede Medellín, Medellín, Colombia
}

\begin{abstract}
Resumen
El estudio biológico y de población del cangrejo rey Damithrax spinosissimus es fundamental para establecer los planes de manejo de esta especie en el Caribe colombiano. Este estudio brinda información sobre las tallas, la proporción entre sexos y las relaciones biométricas de D. spinosissimus en tres islas del Caribe colombiano (Providencia, Rosario y Tintipán), la cual se recogió en muestreos mensuales llevados a cabo entre septiembre de 2011 y marzo de 2012. Se recolectaron 462 ejemplares en las islas. La proporción de sexos fue de 1:1, con 51,5\% de hembras y 48,4 \% de machos. La proporción de hembras ovadas con respecto a las no ovadas también fue de 1:1. El promedio de la longitud del caparazón (LC) fue de 11,6 cm tanto en machos como en hembras, mientras que el ancho del caparazón (AC) fue de 11,9 cm en las hembras y de 12,1 cm en los machos. La longitud promedio del caparazón

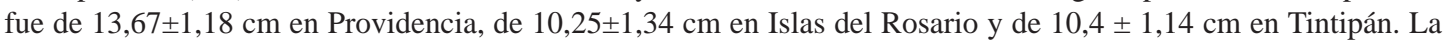
relación entre la longitud y el ancho del caparazón se describió mediante la ecuación LC=-0,393186+1,06755*AC, mientras que la relación entre el peso total $(\mathrm{P})$ y la longitud del caparazón se describió mediante la ecuación Ln P=1,64254+3,36711 Ln LC.
\end{abstract}

Palabras claves: biología, Caribe, cangrejo rey, Damithrax, estructura de poblaciones.

Population structure of the king crab Damithrax spinosissimus in the Colombian Caribbean region

\begin{abstract}
The biological and population study of the king crab Damithrax spinosissimus is essential to establish management plans for this species in the Colombian Caribbean region. This study provides information on size, sex ratio and biometric relationships of $D$. spinosissimus in three Colombian Caribbean islands (Providencia, Rosario and Tintipán), based on monthly surveys conducted between September 2011 and March 2012. A total of 462 individuals were collected. The sex ratio was $1: 1$ with $51.5 \%$ of females and $48.4 \%$ of males. Similarly, the proportion of ovigerous and non-ovigerous females was 1:1. The average shell length (LC) was $11.6 \mathrm{~cm}$ for both males and females, while the width of the shell (AC) was $11.9 \mathrm{~cm}$ for females and $12.1 \mathrm{~cm}$ for males. The average shell length was $13.67 \pm 1.18$ cm in Providence, $10.25 \pm 1.34 \mathrm{~cm}$ in Islas del Rosario and $10.4 \pm 1.14 \mathrm{~cm}$ in Tintipán. The relationship between carapace length and width was described by the equation LC $=-0.393186+1.06755^{*}$ AC, whereas the relationship between the total weight $(\mathrm{P})$ and the carapace length was described by the equation Ln P=-1.64254+3.36711 Ln LC.
\end{abstract}

Key words: Biology, Caribbean, king crab, Damithrax, population structure.

\section{Introducción}

La superfamilia Majoidea presenta una distribución amplia en todos los mares del mundo (Cruz \& Campos, 2000), y una gran diversidad dentro del infraorden Brachyura (Hultgren \& Stachowicz, 2008). Este grupo de crustáceos braquiuros es importante por su papel como consumidores primarios y por su abundante contribución de larvas al zooplancton (Vélez, 1978). En el Caribe colombiano se han registrado aproximadamente 58 especies de Majoidae, sobre la mayoría de las cuales hay grandes vacíos de información en cuanto a los aspectos biológicos y a la estructura de tallas y de la fecundidad, entre otros (Cruz \& Campos, 2000).
Damithrax spinosissimus (Lamarck, 1818) es un cangrejo de gran tamaño que habita en el Caribe, generalmente en aguas someras, hasta los $179 \mathrm{~m}$ de profundidad y en ambientes arrecifales, los cuales utiliza como refugio en muchas ocasiones (Williams, 1984). Su distribución se extiende desde Carolina del Sur, Estados Unidos, el Golfo de México hasta Nicaragua y desde las Antillas hasta Venezuela, incluido Colombia (Rodríguez \& Hendrickx, 1992). En el

\footnotetext{
*Correspondencia:

Néstor Hernando Campos, nhcamposc@unal.edu.co

Recibido: 7 de julio de 2015

Aceptado: 5 de noviembre de 2015
} 
Caribe colombiano se ha recolectado en las Islas del Rosario y de San Bernardo (Bermúdez, et al., 2002). El nombre del género fue cambiado recientemente de Mithrax a Damithrax (Windsor \& Felder, 2014).

Los estudios sobre esta especie en el Caribe se han centrado en su identificación, abundancia y distribución, así como en el desarrollo larval en condiciones controladas (Provenzano \& Brownell, 1977) y en los hábitos de alimentación, pues se la considera una especie con gran potencial en la maricultura (Creswell, et al., 1989). Sin embargo, la información sobre la especie en el Caribe colombiano es escasa en lo relativo a los aspectos biológicos y de poblaciones, pues los estudios se han limitado a la distribución y el registro de las especies en el área. La falta de información sobre la población y la fuerte presión pesquera que sufre esta especie motivó que en el 2002 se la incluyera en el Libro rojo de invertebrados marinos de Colombia en la categoría de "vulnerable”, pero sin mayor información sobre el estado real de la población, al menos en las áreas donde se ha reportado la presencia de la especie (Bermúdez, et al., 2002). Este estudio se propuso aportar, por primera vez en el Caribe colombiano, información sobre las tallas, la proporción entre sexos y las relaciones biométricas de $D$. spinosissimus, con miras a complementar los datos que permitan un manejo integral del recurso y la adopción de decisiones basadas en datos biológicos actualizados.

\section{Materiales y métodos}

El estudio se desarrolló en tres sitios diferentes en el mar Caribe colombiano: 1) Isla de Providencia, 2) Islas del Rosario e 3) Isla Tintipán. La Isla de Providencia está situada a $80 \mathrm{~km}$ al noroeste de San Andrés y a unos $220 \mathrm{~km}$ de Nicaragua. El Archipiélago de Islas del Rosario se encuentra localizado a $46 \mathrm{~km}$ al suroeste de la ciudad de Cartagena de Indias, en la costa norte colombiana, y la Isla Tintipán es la más grande de las islas costeras que conforman el Archipiélago de San Bernardo en el golfo de Morrosquillo, a $50 \mathrm{~km}$ al suroeste de Cartagena de Indias (Figura 1).

Los ejemplares de $D$. spinosissimus se recolectaron mensualmente durante el día en Providencia e Islas del Rosario entre septiembre de 2011 y marzo de 2012, mientras que en Isla Tintipán se recolectaron solo durante los meses de febrero y marzo de 2012, debido a que se desconocía la presencia de esta especie en el área.

Durante el estudio se recolectaron 462 individuos, con un promedio de 42 por mes. Los individuos se capturaron mediante buceo a pulmón libre.

Los ejemplares se separaron por sexos y estadio de desarrollo, teniendo en cuenta la forma del abdomen y su grado de adherencia a la cavidad ventral del tórax según el criterio empleado para la especie Mithraculus forceps (Hernández-

Reyes, et al., 2001). Los machos se agruparon en juveniles y adultos, mientras que las hembras se clasificaron en hembras juveniles, hembras adultas y hembras ovadas. Además, a cada ejemplar se le midió la longitud (LC) y el ancho del caparazón (AC) con ayuda de un calibrador, y se pesaron (P) y fotografiaron (cámara Canon) para luego devolverlos vivos al medio.

Con los datos de las tallas se hicieron diagramas de distribución de frecuencias y comparaciones entre las distribuciones mediante la prueba de Kolmogorov-Smirnov, y también se aplicaron análisis estadísticos descriptivos para observar el comportamiento de las variables. Las variaciones entre las categorías sexuales, espaciales y temporales de la variable LC se compararon mediante el análisis de la varianza (ANOVA) de una vía para cada categoría (Sokal \& Rohlf, 1995). Los supuestos de normalidad y homogeneidad de varianzas se comprobaron mediante la gráfica de los residuos, y con el test de Shapiro-Wilks y la prueba de Levene, respectivamente (Sokal \& Rohlf, 1995). Para comparar la longitud del caparazón (LC) por sexos, y espacial y temporalmente, se aplicó el test no paramétrico de Kruskall-Wallis debido a que no se cumplieron los supuestos de normalidad y homogeneidad de varianzas ni siquiera después de la transformación de los datos.

Para determinar la proporción entre sexos en el Caribe colombiano en las localidades y en función del tamaño, se empleó la ecuación establecida por Creasey, et al. (1997):

$$
\mathrm{S} 0=(\mathrm{M}-\mathrm{H}) /(\mathrm{M}+\mathrm{H}),
$$

dónde $\mathrm{M}$ corresponde al número de machos en la muestra y $\mathrm{H}$ al número de hembras en la muestra; los valores cercanos a 0 indican proporciones iguales, los valores negativos indican más hembras que machos y los valores positivos, más machos que hembras.

Con el fin de establecer las diferencias en las proporciones entre machos y hembras, y entre hembras ovadas y el total de hembras, se utilizó el estadístico de chi al cuadrado, tomando como proporciones esperadas 1:1 y 0,5:1 (MerchánCepeda, et al., 2009).

La relación entre las variables de longitud y ancho del caparazón se determinó mediante el análisis de regresión lineal descrito por las siguientes ecuaciones (Arana, 2000):

$$
\mathrm{LC}=\mathrm{a}+\mathrm{b} * \text { AC y AC }=\mathrm{a}+\mathrm{b} * \mathrm{LC},
$$

donde LC corresponde a la longitud del caparazón en cm y AC a su ancho, también en cm; a es la constante de regresión, y b, el coeficiente de la regresión.

La determinación de la relación entre longitud y peso (determinación del crecimiento alométrico), se hizo mediante la determinación de la constante de alometría b para cada relación:

$$
\mathrm{P}=\mathrm{a} * A C^{\mathrm{b}} \mathrm{y} \mathrm{P}=\mathrm{a}^{*} \mathrm{LC}^{\mathrm{b}},
$$

donde $\mathrm{P}$ es el peso total en g, LC es la longitud del caparazón en cm, AC su ancho en cm y a y b son los parámetros de la regresión. Se empleó también la linealización del modelo:

$$
\ln \mathrm{P}=\mathrm{a}+\mathrm{b} \ln \mathrm{AC} \mathrm{y} \ln \mathrm{P}=\mathrm{a}+\mathrm{b} \ln \mathrm{LC},
$$




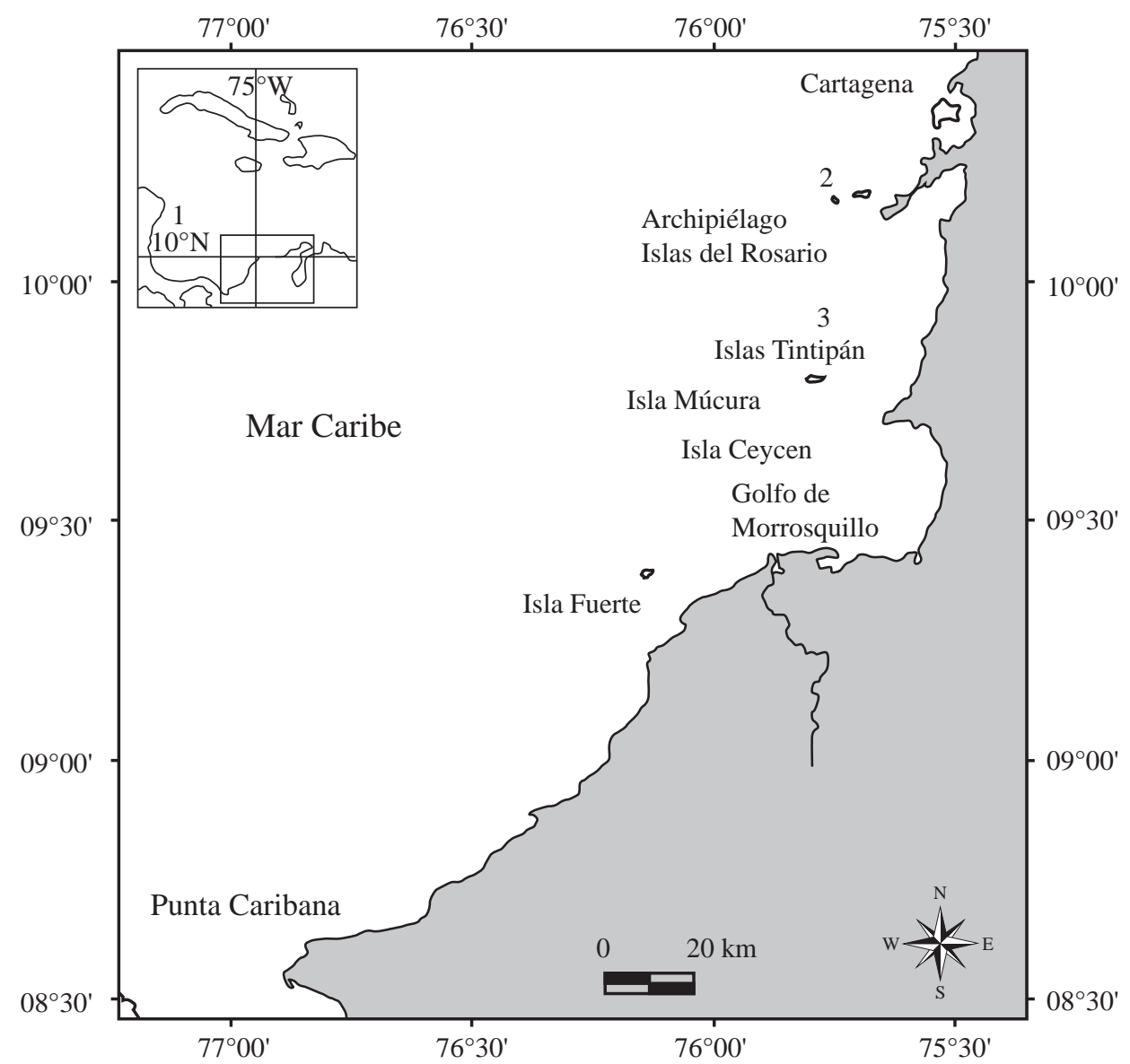

Figura 1. Localización de los sitios de muestreo en el Caribe colombiano, de ejemplares de Damithrax spinosissimus: 1 Isla de Providencia. 2 Islas del Rosario. 3 Isla Tintipán

con lo que los valores de b mayores a 3 indicaban un crecimiento positivamente alométrico, los menores a 3 , un crecimiento alométrico negativo, y los iguales a 3, una condición de isometría (Huxley, 1950; Pinheiro \& Fiscarelli, 2009).

\section{Resultados}

En la tabla 1 se presenta el número de individuos recolectados por sitio de muestreo y clasificados por sexos y estado de desarrollo. En Providencia se recolectó el mayor número de ejemplares, con una mayor proporción de hembras (126), seguido por Islas del Rosario con 76 e Isla Tintipán con 36. Por el contrario, el mayor número de machos se capturó en Islas del Rosario, con 109 ejemplares. Del total de ejemplares recolectados, el mayor porcentaje correspondió a los machos adultos de Islas del Rosario, seguido por las hembras ovadas de Providencia. También en Islas del Rosario se capturó el mayor número de ejemplares inmaduros: 16 machos y 12 hembras juveniles. En todas las estaciones el mayor número de ejemplares recolectados correspondió a los machos adultos, con 201, sin embargo, al agruparlos por sexos, e independientemente del grado de desarrollo, el número fue ligeramente mayor para las hembras (238), lo que indica una leve diferencia de la proporción 1:1 $\left(X^{2}=0,21 ; p>0,05\right.$, ecuación de Creasey=-0,03).

\section{Estructura de las poblaciones}

De los 462 ejemplares de $D$. spinosissimus analizados, $51,50 \%$ correspondió a hembras y $48,40 \%$ a machos. En promedio, las hembras y los machos presentaron valores similares con respecto a la longitud, mientras que el ancho y el peso en los machos mostró un valor promedio mayor que en las hembras, lo que evidencia un leve dimorfismo sexual con respecto a estas variables (Tabla 2).

La distribución de frecuencias de tallas para el total de los individuos recolectados en el Caribe colombiano no presentó una distribución normal (Kolmogorov-Smirnov: $\mathrm{P}=0,000 ; \mathrm{p}<0,05)$. La longitud promedio del caparazón de los ejemplares de $D$. spinosissimus fue de $11,64 \pm 2,09 \mathrm{~cm}$ y el rango de la longitud se situó entre 5,4 y $16 \mathrm{~cm}$; sin embargo, la mayoría de individuos presentaron tallas entre los 9 y $15 \mathrm{~cm}$, siendo pocos los individuos de tallas pequeñas. Con respecto al ancho del caparazón, el promedio estuvo en $12,01 \pm 2,80 \mathrm{~cm}$, mientras que el rango estuvo entre 1,5y11,71

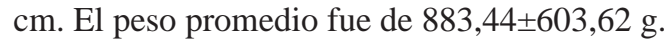


Tabla 1. Número de ejemplares de Damithrax spinosissimus recolectados, por lugar de muestreo y grupos de desarrollo.

\begin{tabular}{lrrrrrrrr}
\hline & \multicolumn{2}{c}{ Providencia } & \multicolumn{2}{c}{ I. Rosario } & \multicolumn{2}{c}{ I. Tintipán } & \multirow{2}{*}{ Total } & \% \\
& Total & \% & Total & \% & Total & \% & 7,0 & 23 \\
\hline Machos juveniles & 1 & 0,5 & 16 & 8,6 & 6 & 5,0 \\
Machos adultos & 64 & 33,5 & 93 & 50,3 & 44 & 51,2 & 201 & 43,5 \\
Hembras juveniles & 1 & 0,5 & 12 & 6,5 & - & - & 13 \\
Hembras adultas & 35 & 18,3 & 33 & 17,8 & 13 & 15,1 & 81 \\
Hembras ovadas & 90 & 47,1 & 31 & 16,8 & 23 & 26,7 & 14,5 \\
Total & 191 & & 185 & & 86 & 31,2 \\
\hline
\end{tabular}

Tabla 2. Valores promedio por sexos de la longitud (LC) y ancho del caparazón (AC) en centímetros y peso total (P) en gramos de Damithrax spinosissimus en el Caribe colombiano.

\begin{tabular}{ccrrcc}
\hline Sexo & Variable & \multicolumn{1}{c}{$\mathbf{X}$} & \multicolumn{1}{c}{ SD } & Máximo & Mínimo \\
\hline \multirow{4}{*}{ Hembras } & LC (cm) & 11,6 & 2,06 & 15,5 & 6,5 \\
\cline { 2 - 6 } & AC (cm) & 11,9 & 2,24 & 17,1 & 6,5 \\
& P (gr) & 840,1 & 526,90 & 3100 & 110 \\
\multirow{4}{*}{ Machos } & LC(cm) & 11,6 & 2,13 & 16 & 5,4 \\
& AC (cm) & 12,1 & 2,32 & 17 & 6,2 \\
& P (gr) & 929,5 & 673,92 & 3800 & 1200 \\
\hline
\end{tabular}

\section{Tallas por sexo}

La distribución de la frecuencia de las tallas de los sexos combinados con respecto a la longitud del caparazón en los sitios de muestreo, no fue normal (Kolmogorov-Smirnov: $\mathrm{P}=0,000 ; \mathrm{p}<0,05$ ) (Figura 2). Se destaca que las frecuencias en las que se presentó el mayor número de individuos fue similar en los dos sexos (9,3-10,0 y 11,3-12,0 cm), con excepción de la frecuencia de 13,3-14,0 cm, que fue más abundante en las hembras.

La mayoría de las frecuencias de talla fue siempre superior en las hembras adultas (ovadas y no), y en algunos casos duplicaron el número de machos (Figura 2). Por el contrario, en las tallas más pequeñas solo se recolectaron machos inmaduros $(<6 \mathrm{~cm})$.

A pesar de estos resultados y en lo tocante a la distribución por sexos, no se encontraron diferencias significativas entre la longitud promedio del caparazón de las hembras $(11,68 \pm 2,06 \mathrm{~cm})$ y de los machos adultos $(11,62 \pm 2,12 \mathrm{~cm})$ (Kruskall-Wallis: $\mathrm{P}=0,815 ; \mathrm{p}>0,05$ ). Sin embargo, cuando se compararon hembras y machos juveniles se encontraron diferencias significativas en la longitud del caparazón (ANOVA: $\mathrm{P}=0,029 ; \mathrm{p}<0,05$ ) (Tabla 3).

\section{Estructuras espacial y temporal de tallas en el Caribe colombiano}

La distribución mensual de las tallas del cangrejo D. spinosissimus para el Caribe colombiano (Figura 3) varió entre 5,5 y

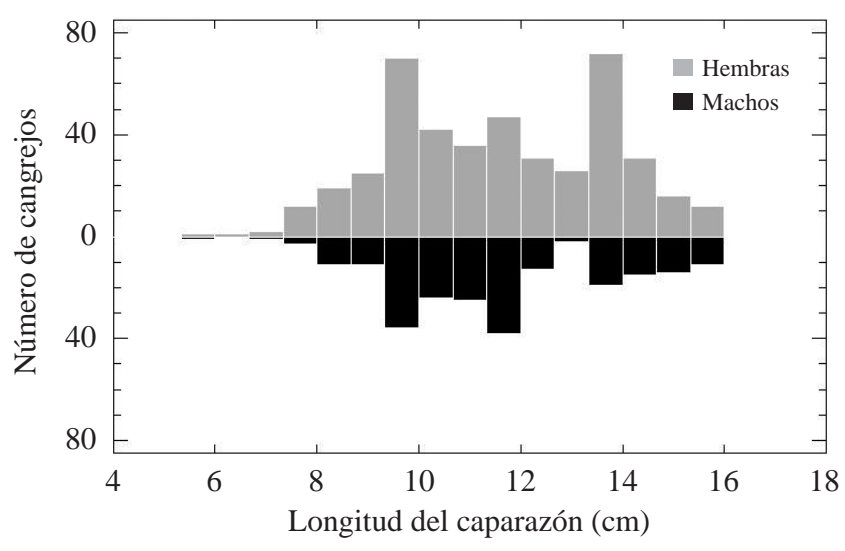

Figura 2. Distribución de frecuencia tallas por sexos de Damithrax spinosissimus en el Caribe colombiano.

15,5 cm, rango que se encuentra con frecuencia; sin embargo, en todos los meses fueron más abundantes las tallas grandes, en el rango de 10,0 a 14,5 cm. Las mayores tallas promedio se presentaron en los meses de septiembre $(12,36 \pm 1,54$ cm) y octubre $(12,96 \pm 1,96 \mathrm{~cm})$ de 2011 y de enero de 2012 $(12,03 \pm 2,17 \mathrm{~cm})$, mientras que en los meses de febrero $(11 \pm 2,05 \mathrm{~cm})$ y marzo $(11,13 \pm 1,92 \mathrm{~cm})$ de 2012 se presentaron menores longitudes promedio de caparazón debido, probablemente, al efecto de las condiciones climáticas de la época (influencia de los vientos alisios) en las poblaciones, lo que provoca migraciones a zonas más profundas en busca de condiciones más estables y de menor influencia de los vientos.

El valor promedio de las tallas aumentó en los meses finales de la época de lluvias y comienzo de la época seca, registrándose un aumento de tallas con el incremento de la descarga de aguas de escorrentía, lo que posiblemente favoreció una mayor disponibilidad de nutrientes y un crecimiento de los tapetes algales (Figura 3).

\section{Tallas por localidad}

Al comparar las tallas promedio de $D$. spinosissimus entre las localidades, se presentaron diferencias estadísticamente significativas entre los ejemplares de Providencia y los de Islas de Rosario y Tintipán (Kruskall-Wallis: $\mathrm{P}=0,00$; $\mathrm{p}<0,05$ ) (Figura 4). 
En el Tabla 4 se presentan los resultados de la comparación entre las tres áreas de muestreo. De acuerdo a estos resultados, hubo diferencias notorias en la estructura de las tallas entre Providencia y las poblaciones de Islas del Rosario y de la Isla de Tintipán.

En la Figura 5 se muestra la distribución de frecuencias de la longitud del caparazón del cangrejo rey del Caribe por sitio de muestreo. Las frecuencias de tallas no se ajustaron a distribuciones normales en las poblaciones de Providencia

Tabla 3. Información de ANOVA para la comparación entre machos y hembras juveniles de Damithrax spinosissimus en el Caribe colombiano.

\begin{tabular}{ccrlll}
\hline Fuente & $\begin{array}{c}\text { Suma de } \\
\text { Cuadrados }\end{array}$ & Gl & $\begin{array}{c}\text { Cuadrado } \\
\text { Medio }\end{array}$ & Razón-F & Valor-P \\
\hline Entre grupos & 3,7582 & 1 & 3,7582 & 5,17 & 0,0298 \\
\hline Intra grupos & 23,2594 & 32 & 0,726858 & & \\
\hline Total (Corr.) & 27,0176 & 33 & & & \\
\hline
\end{tabular}

(Kolmogorov-Smirnov: $\mathrm{P}=0,021 ; \mathrm{p}<0,05$ ), de Islas del Rosario (Kolmogorov-Smirnov: $\mathrm{P}=0,007$; $\mathrm{p}<0,050$ ) y Tintipán (Kolmogorv-Smirnov: $\mathrm{P}=0,018$; $\mathrm{p}<0,05$ ). En Providencia la mayoría de los ejemplares recolectados presentaron tallas superiores a $10 \mathrm{~cm}$, en Islas del Rosario las mayores frecuencias correspondieron a las tallas intermedias (<10 y $12 \mathrm{~cm}$ ) y la talla máxima estuvo bien representada. Por último, en Tintipán predominaron las tallas menores.

En Providencia la longitud promedio del caparazón de los

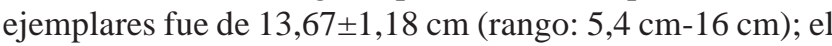
ancho promedio del caparazón fue de 14,20 $\pm 1,32 \mathrm{~cm}$ (rango: 5,4-17,1 cm); el peso promedio fue de 1.434,13 $\pm 560,46 \mathrm{~g}$. En Islas del Rosario la longitud promedio del caparazón de los individuos fue de $10,25 \pm 1,34 \mathrm{~cm}$, el ancho promedio del caparazón fue de 10,56 $\pm 1,58$, mientras que el peso promedio de los individuos fue de 502,27 $\pm 213,14$ g. Por último, la talla promedio de los ejemplares recolectados en Tintipán fue de $10,11 \pm 0,97 \mathrm{~cm}(8,0-12,5 \mathrm{~cm})$ y el peso promedio fue de 493,14 $\pm 173,41 \mathrm{~g}$.
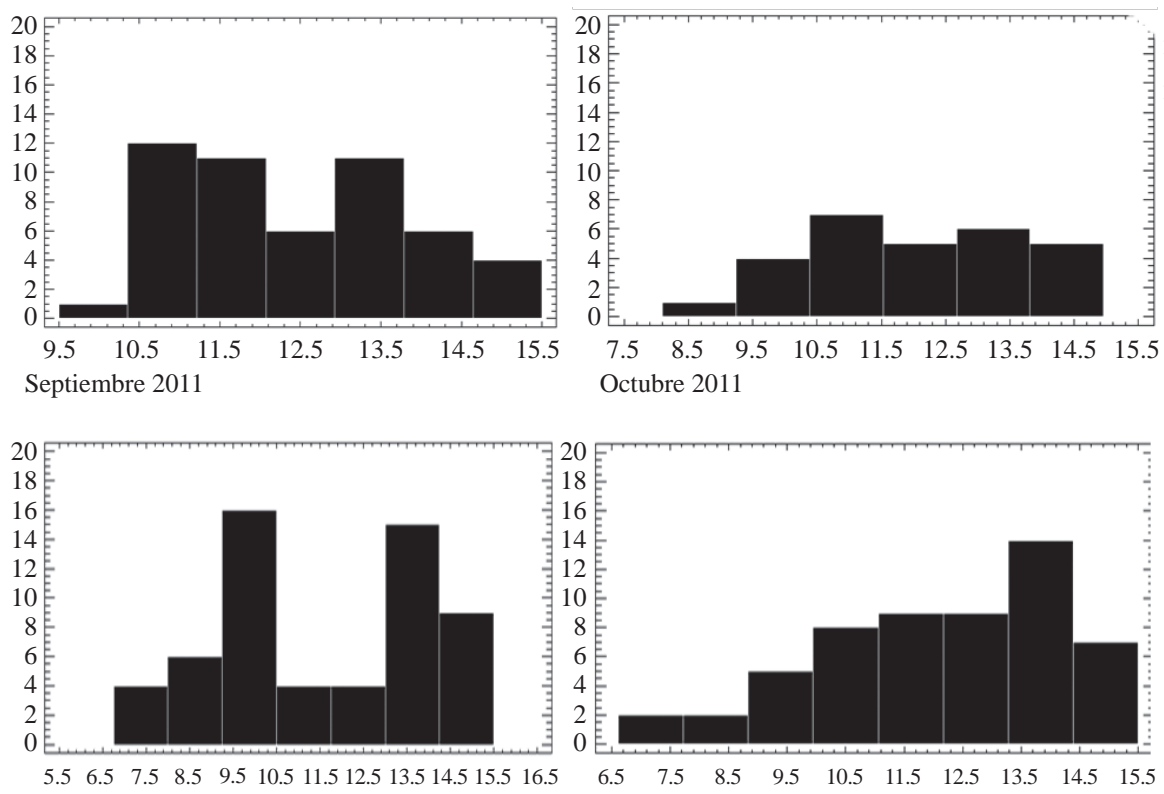
Diciembre 2011
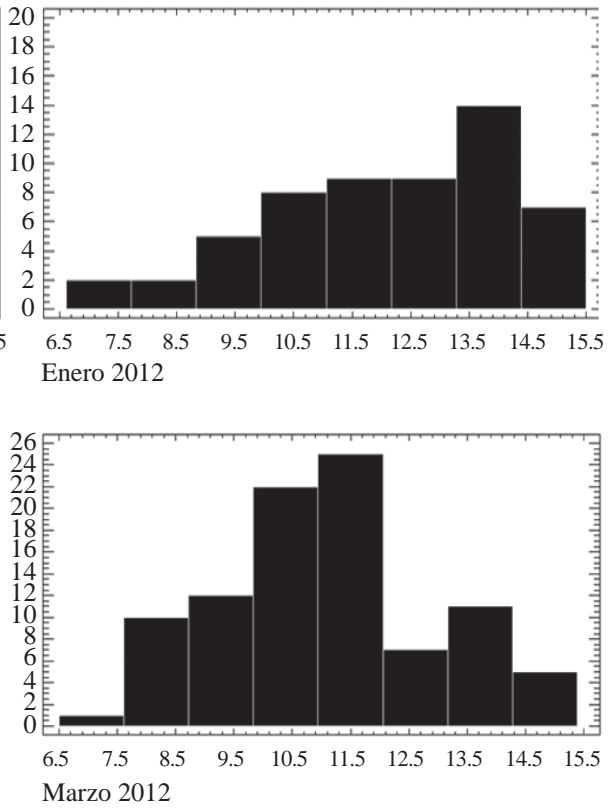

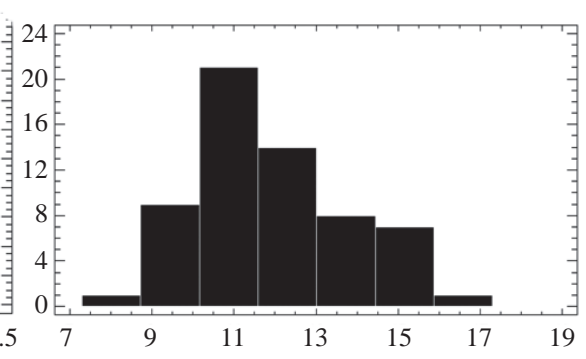

Noviembre 2011

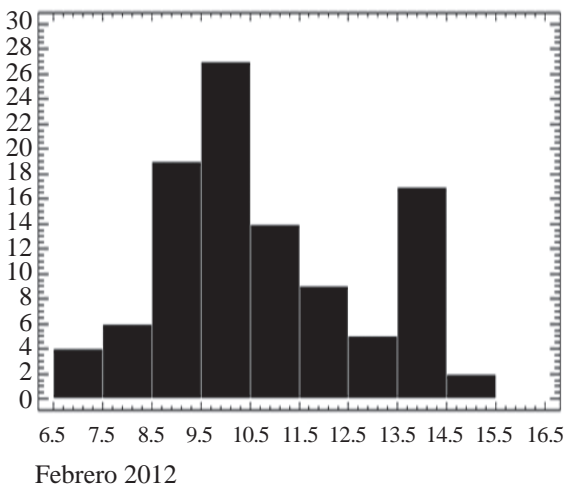

Longitud del caparazón

Figura 3. Distribución mensual de frecuencias de tallas en machos de cangrejo rey del Caribe, septiembre 2011 a marzo 2012. 
Box-and-Whisker Plot

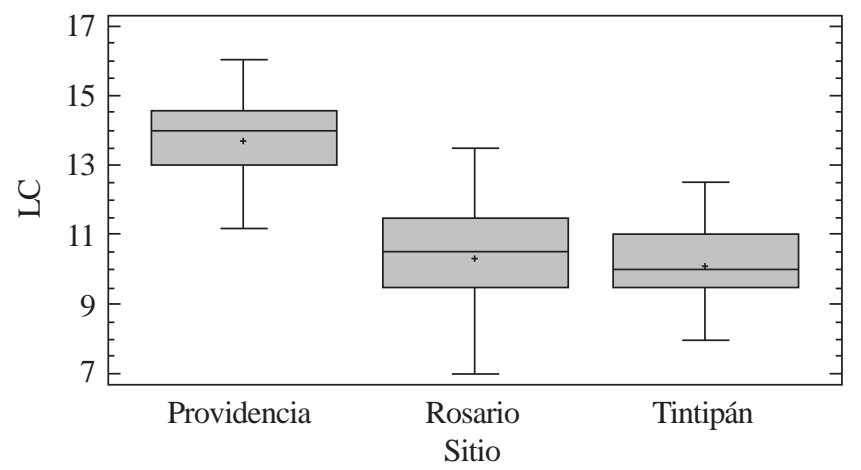

Figura 4. Comparación de la Longitud del caparazón entre las áreas muestreadas en el Caribe colombiano.

Tabla 4. Comparación entre las poblaciones del cangrejo rey del Caribe, Damithrax spinosissimus, en las tres localidades de muestreo (P): Providencia, (R): Rosario y (T): Tintipán, de acuerdo a la prueba de múltiples rangos LSD.(*): Indica una diferencia.

\begin{tabular}{cccc}
\hline Contraste & Sig. & Diferencia & +/- Límites \\
\hline P - R & $*$ & 3,26736 & 0,336596 \\
P - T & $*$ & 3,7222 & 0,378654 \\
R - T & $*$ & 0,454839 & 0,450616 \\
\hline
\end{tabular}

Al comparar la longitud del caparazón por sexo se observaron diferencias significativas entre hembras y machos en Providencia (Kruskall-Wallis: $\mathrm{P}=0,00 ; \mathrm{p}<0,05$ ) (Figura 6A); en Islas del Rosario (Kruskall-Wallis: $\mathrm{P}=0,00 ; \mathrm{p}<0,05$ ) (Figura 6B) y en Tintipán (ANOVA: $\mathrm{P}=0,001 ; \mathrm{p}<0,05$ ) (Figura 6C). En las tres localidades la longitud del caparazón de los machos (Providencia: 10,6-16 cm; Islas del Rosario: 7,3-13,5 cm; Tintipán: 10,44 $\pm 0,97 \mathrm{~cm}$ ) fue mayor que el de las hembras (Providencia 10,69-15,5 cm; Islas del Rosario:

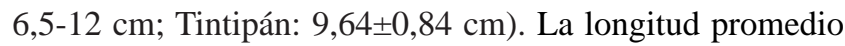

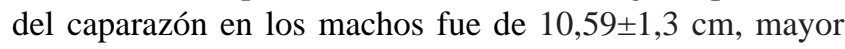
que las tallas de las hembras $(9,57 \pm 1,37 \mathrm{~cm})$.

\section{Relación entre la longitud y el ancho del caparazón}

Se determinó la relación entre la longitud y el ancho del caparazón. En la Figura 7 se presenta la relación para el total de los individuos analizados (A), separados por sexos (B y C), y se incluyen las ecuaciones utilizadas para describirla, así como el coeficiente de correlación.

El análisis se ajustó a una regresión lineal, lo cual confirmó la relación de las dos variables en la población total o separada por sexos, por lo que solo se tuvo en cuenta la longitud para los análisis. D. spinosissimus presentó una relación estadísticamente significativa entre la longitud y el ancho del caparazón, con un coeficiente de correlación de 0,98 y un coeficiente de determinación $\left(\mathrm{R}^{2}\right)$ de $96,1 \%$ (Figura 7A).
A

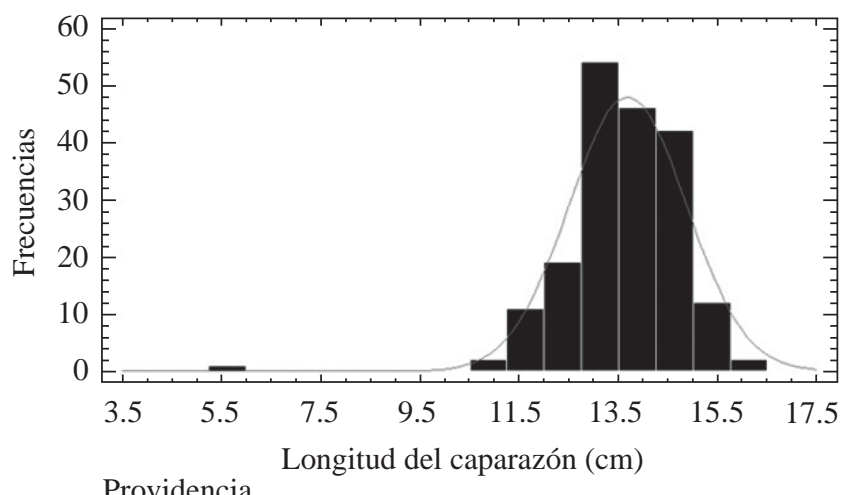

Providencia
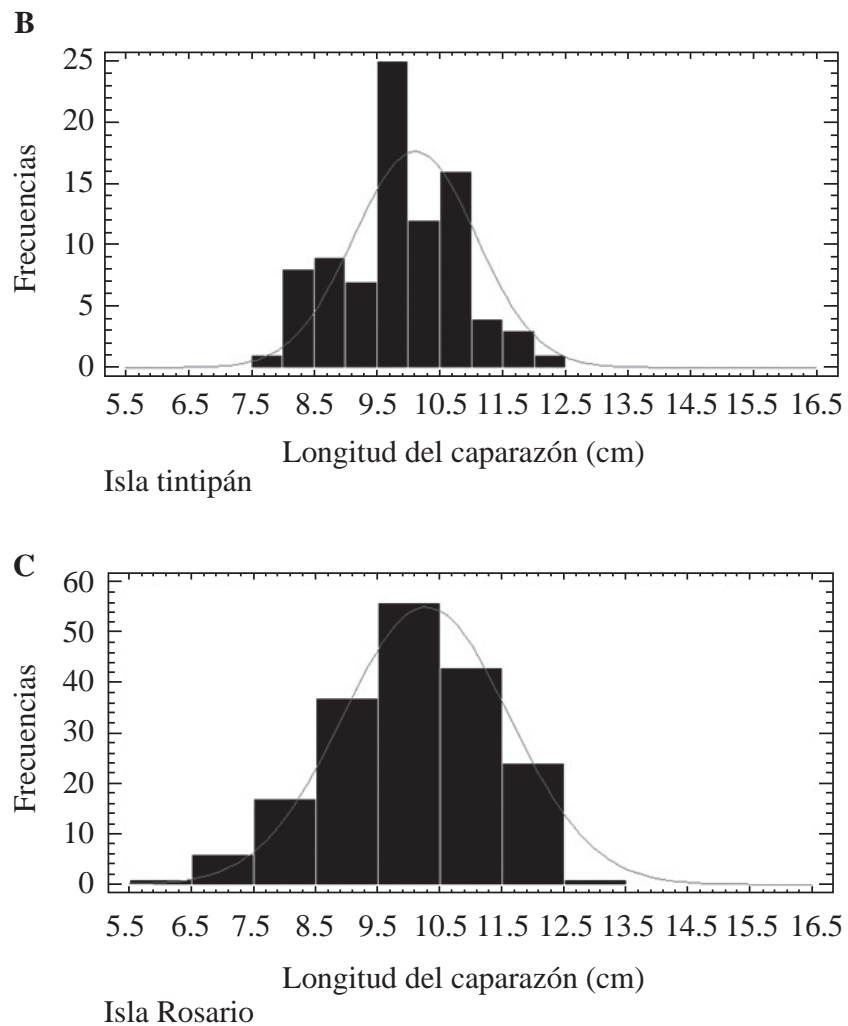

Figura 5. Distribución de frecuencias de la longitud del caparazón de cada una de las localidades muestreadas. A. Providencia, B. Islas del Rosario C. Isla Tintipán.

La relación entre la longitud y el ancho del caparazón para hembras y machos fue similar, con valores de $\mathrm{R}^{2}$ de 95,6 y $95,9 \%$, respectivamente, y una relación significativa entre las variables $(\mathrm{P}=0,000 ; \mathrm{p}<0,05)$ en hembras y machos (Figuras 7B y C).

La relación de LC y AC de $D$. spinosissimus por sitio de muestreo fue directa $(\mathrm{P}=0,000 ; \mathrm{p}<0,05)$. Para Providencia se obtuvo la siguiente ecuación: $\mathrm{AC}=0,056+1,0323 * \mathrm{LC}$, y un coeficiente de correlación de 0,91 ; para Islas del Rosario la ecuación que describe la relación de las dos variables fue: $\mathrm{AC}=-1,1698+1,1433^{*} \mathrm{LC}$, y el coeficiente de 
A

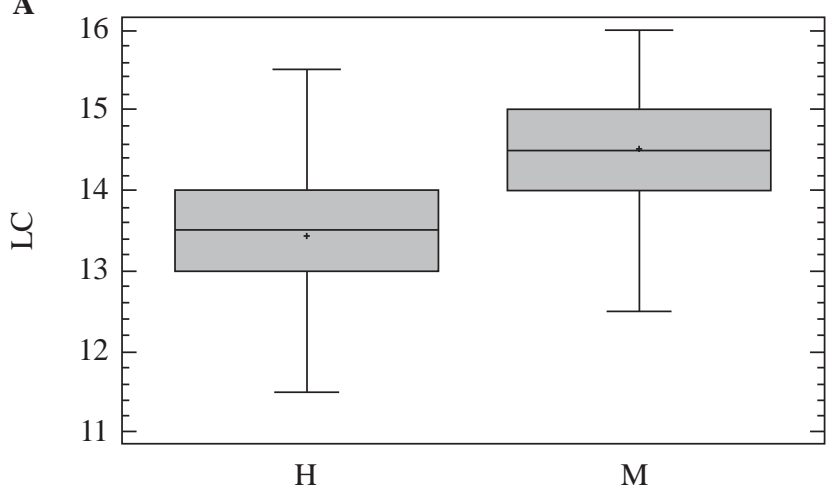

B
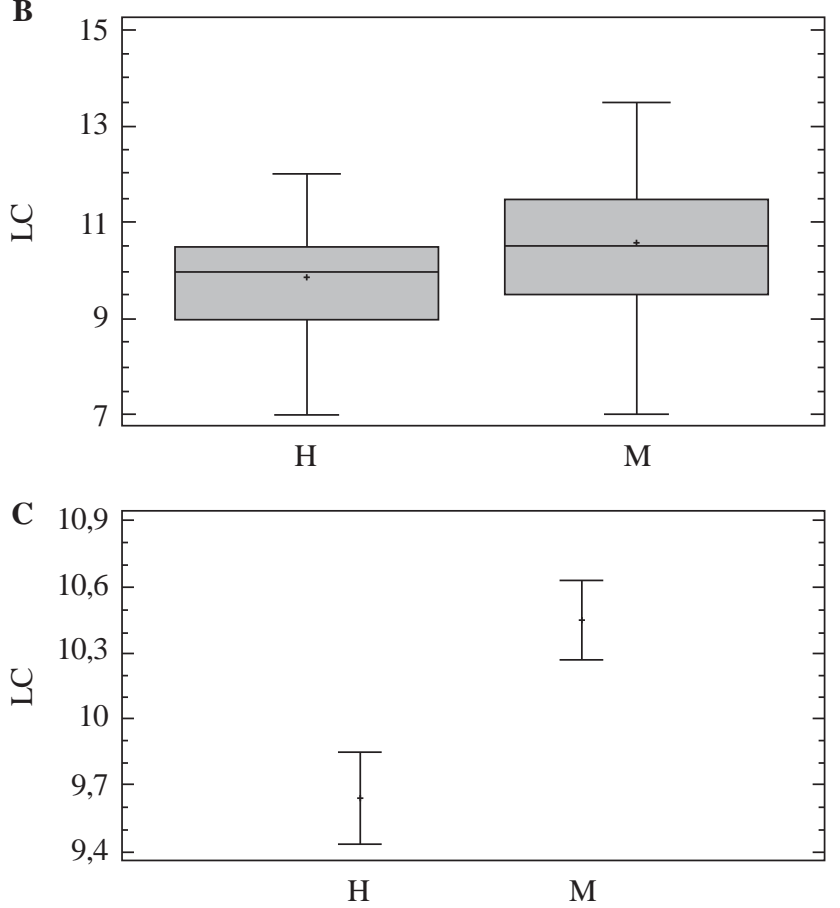

Figura 6. Longitud promedio, desviación estándar y valores máximos y mínimos para los ejemplares de Damithrax spinosissimus recolectados en . A) Providencia comparación entre machos (M) y hembra. B) Islas del Rosario Comparación entre machos (M) y hembras (H). C) Isla Tintipán Comparación entre Machos (M) y hembras $(\mathrm{H})$.

correlación, 0,97; en Tintipán la relación se describió así: $\mathrm{AC}=-0,8007+1,1137 * \mathrm{LC}$, y el coeficiente de correlación fue de 0,94 .

El valor de la pendiente en la población de Providencia se diferenció ligeramente de los otros dos sitios de muestreo y se asemejó al valor obtenido para el total de los individuos.

En la Tabla 4 se presentan los resultados del análisis de regresión para las variables de longitud y ancho del caparazón separado por sexos. En Providencia se observó una relación estadísticamente significativa entre machos y hembras $(\mathrm{P}=0,000 ; \mathrm{p}<0,05)$; sin embargo, en las hembras la relación entre las variables fue menos determinante, dado que el valor de $\mathrm{R}^{2}$ fue de $71,5 \%$ y el coeficiente de regresión (CR) fue de 0,84. En contraste, en los machos la relación fue más determinante, con un $\mathrm{R}^{2}$ de 89,3 \% y un coeficiente de regresión de 0,94 . El valor de la pendiente fue ligeramente menor en las hembras que en los machos, lo que probablemente se debió a que estos no detienen el crecimiento, como sucede en muchos crustáceos, mientras que una vez que maduran, las hembras no mudan más.

En las Islas del Rosario la relación entre la longitud y el ancho del caparazón presentó una relación estadísticamente significativa $(\mathrm{P}=0,000 ; \mathrm{p}<0,05)$ en los dos sexos. En este caso, los dos sexos presentaron un comportamiento semejante, con un CR de 0,96 para machos y de 0,97 para las hembras, mientras que el $\mathrm{R}^{2}$ fue mayor en las hembras. Igualmente, tanto machos $\left(\mathrm{P}=0,000 ; \mathrm{p}<0,05 ; \mathrm{R}^{2}=95,4 \%\right.$; $\mathrm{CR}=$
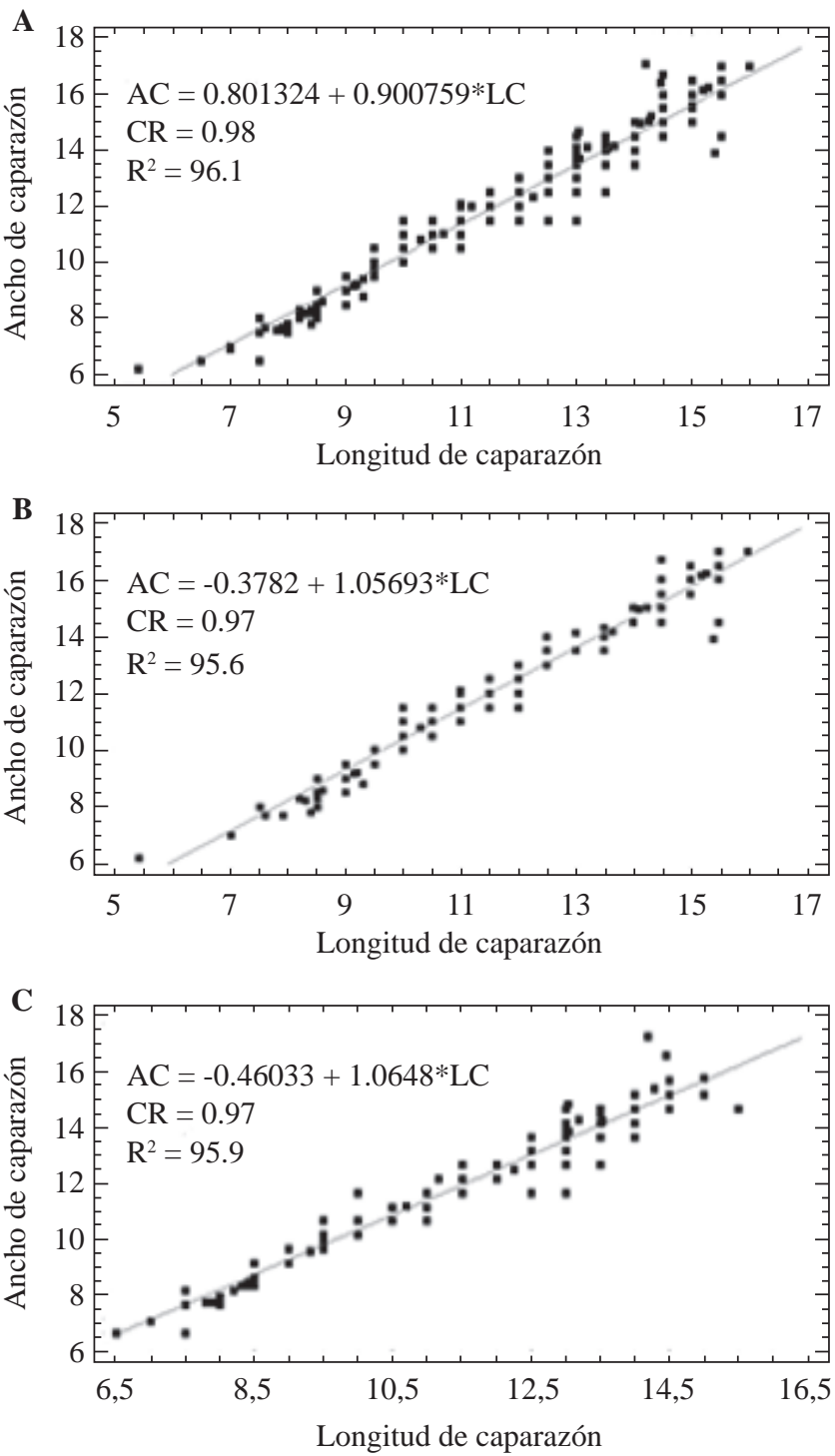

Figura 7. Análisis de regresión para las variables longitud y ancho del caparazón en Damithrax spinosissimus. (A) número total de ejemplares. (B) Machos (C) Hembras. 
0,97) como hembras presentaron una fuerte relación entre las variables, la cual fue estadísticamente significativa $\left(\mathrm{P}=0,000: \mathrm{p}<0,05 ; \mathrm{R}^{2}=93,2 \% ; \mathrm{CR}=0,96\right)$. En este caso, al igual que en Providencia, la pendiente fue mayor en los machos.

Contrariamente a lo observado en los ejemplares recolectados en Providencia e Islas del Rosario, en los ejemplares de Isla Tintipán se obtuvieron mayores valores para las hembras, y los CR fueron de 0,96 y 0,92 para hembras y machos, respectivamente $\left(R^{2}=93,5\right.$ en hembras y $85,1 \%$ en machos). La pendiente fue ligeramente mayor en las hembras $(1,06)$ que en los machos (1,02).

\section{Relación entre la talla y el peso}

Por último, se halló la relación entre el peso y la longitud del caparazón para todos los ejemplares (Figuras 8 A, B) y para los sexos por separado (Figura 8 C, D). La relación se ciñó a una correlación potencial, y en los tres casos la pendiente fue mayor a 3 , lo que denota un crecimiento alométrico positivo, con un CR de 0,93 y un $\mathrm{R}^{2}$ de 87,9 para la longitud y de 89,4 para el ancho. Teniendo en cuenta esta diferencia mínima entre las correlaciones de la longitud y el ancho con el peso, así como los resultados obtenidos en la relación entre longitud y ancho, se decidió continuar con los análisis utilizando solamente el ancho.

A

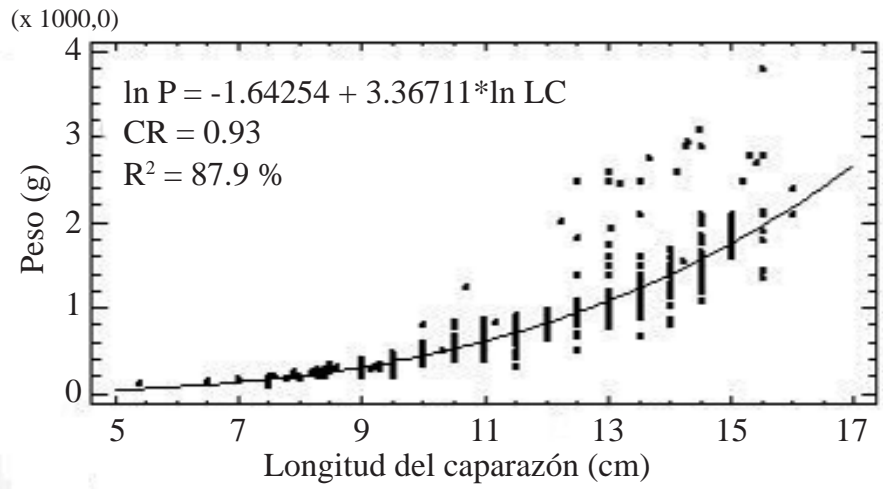

B

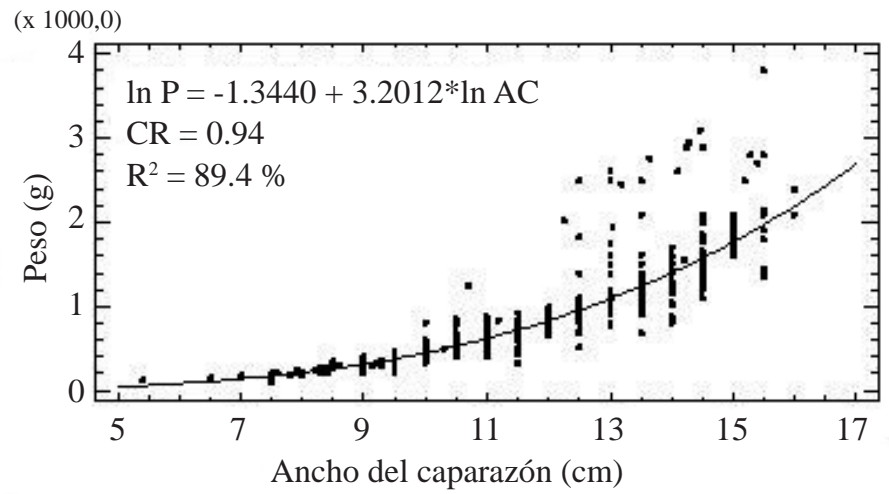

La relación entre peso y ancho del caparazón para machos y hembras por separado tuvo un comportamiento semejante para los dos sexos, con un mismo CR $(0,94)$. En los machos el valor de $\mathrm{R}^{2}$ fue ligeramente mayor (88,9 para las hembras y $90 \%$ para los machos). Igualmente, la pendiente fue ligeramente mayor en los machos (3,15 en hembras y 3,25 en machos), lo que denota en los dos casos un crecimiento alométrico positivo. Estas diferencias se debieron probablemente a que las hembras detienen su crecimiento al alcanzar la madurez sexual y los machos presentan quelas de mayor tamaño, lo que hace que alcancen pesos mayores (Figura 8 C, D).

En el Tabla 5 se presentan los resultados de la correlación entre el ancho del caparazón y el peso del total de los ejemplares por sitio de muestreo y por sexos.

La ecuación que describe la relación $\mathrm{P} V s$. AC de $D$. spinosissimus por sitio de muestreo fue positiva $(\mathrm{P}=0,000$; $\mathrm{p}<0,05)$. Para Providencia se obtuvo la siguiente ecuación: In $\mathrm{P}=-0,531709+2,91839 \ln \mathrm{AC}$, y un coeficiente de correlación de 0,76; para Islas del Rosario, la ecuación que describe la relación de las dos variables fue: $\ln \mathrm{P}=-0,0665353+\ln 2,63793$ AC, y un coeficiente de correlación de 0,92; en Tintipán la relación se describió así: ln P=-1,06221+ 3,07535 ln AC, y el coeficiente de correlación fue de 0,94 (Tabla 6).

\section{C}

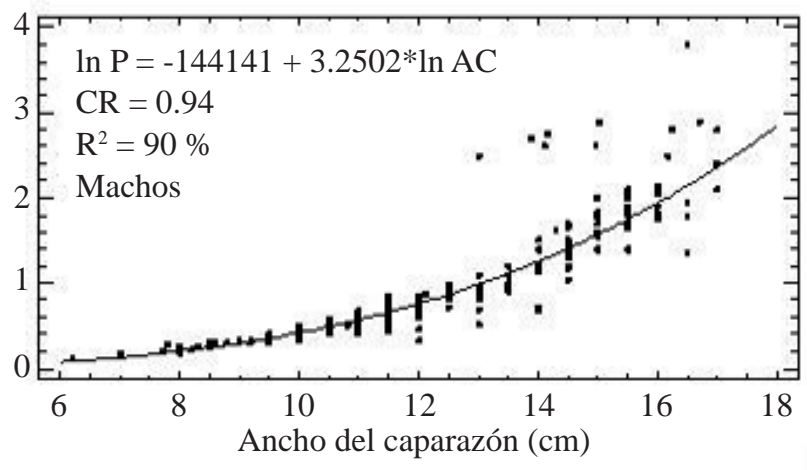

D

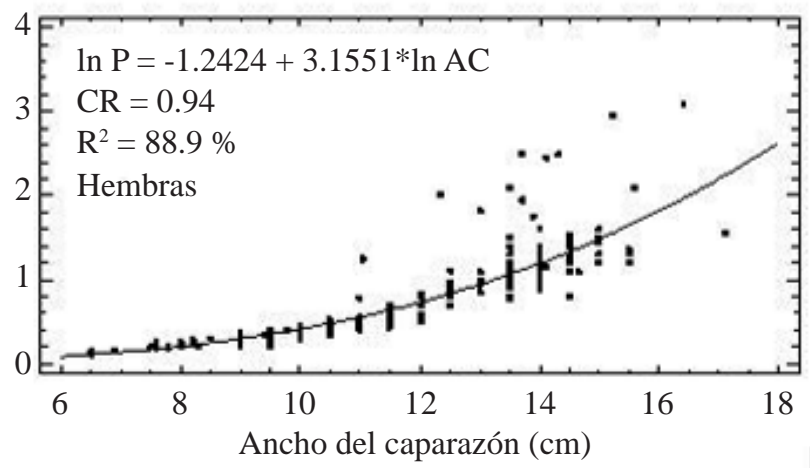

Figura 8. Análisis de regresión para el total de individuos de Damithrax spinosissimus recolectados, de las variables longitud del caparazón vs. peso (A). Ancho del caparazón vs. peso (B). Ancho del caparazón vs. peso en machos (C). Ancho del caparazón vs. peso en hembras (D). 
La pendiente (b) para describir la relación en Providencia y en Islas del Rosario fue menor a 3, lo que denota un crecimiento alométrico negativo, mientras que en Isla Tintipán el valor fue mayor a 3, e indica un crecimiento alométrico positivo.

En cuanto a la relación por sexos, en Providencia se observó que tanto en machos como en hembras la pendiente fue menor a 3, lo que significa un crecimiento alométrico negativo. En Islas del Rosario, por el contrario, se presentó una relación significativa entre las tallas y el peso, tanto para las poblaciones en general como para individuos machos y hembras, con valores de $\mathrm{R}^{2}$ mayores a $80 \%$ y coeficientes de correlación de más de 0,9; de acuerdo a la pendiente, en los tres casos se evidenció un crecimiento alométrico negativo (minorante) (Tabla 6).

Con respecto a la Isla Tintipán, en el total de individuos la relación entre el ancho del caparazón y el peso fue significativa $\left(\mathrm{R}^{2}=89,5 \%, \mathrm{CR}=0,94\right)$, al igual que al calcularla por separado en machos y hembras. Para el total de individuos y para hembras, el valor de la pendiente (b) fue mayor a 3, lo que denota un crecimiento alométrico positivo, mientras que en los machos fue ligeramente menor a 3, por lo que su crecimiento fue alométrico negativo. Muy probablemente, la evidencia de un crecimiento alométrico negativo esté relacionada con la explotación por parte de pescadores artesanales, ya que extraen principalmente los machos por presentar un mayor tamaño (Tabla 6).

\section{Discusión}

La distribución de las frecuencias de las tallas de machos y hembras de $D$. spinosissimus en el Caribe colombiano evidencia tamaños promedio mayores que los registrados en Bocas del Toro, Panamá (Guzmán \& Tewfik, 2004) y en los Cayos de la Florida (Baeza, et al., 2012). En estos trabajos se registró un promedio para los Cayos de 6,54 cm en los machos y de $5,75 \mathrm{~cm}$ en las hembras, mientras que para Bocas del Toro el promedio fue de 9,2 cm en el AC; en el presente estudio los promedios registrados fueron de $11,5 \mathrm{~cm}$ en las hembras y de 12,9 cm en los machos. Estas diferencias en las tallas en algunos braquiuros están asociadas con las variaciones latitudinales (Jones \& Simmons, 1983), y en varios casos la presencia de tallas menores también puede estar determinada por el incremento de la explotación del recurso como consecuencia de la reducción de otras especies altamente comerciales en el Caribe (Tewfik \& Guzmán, 2003).

Tabla 5. Análisis de regresión para las variables longitud (LC) y ancho del caparazón (AC) en Damithrax spinosissimus, por localidades y sexos (M) Machos (H) Hembras. Se da el coeficiente de correlación (CR), el valor de la pendiente (b) y el coeficiente de determinación ( $\mathrm{R}^{2}$ \%).

\begin{tabular}{|c|c|c|c|c|c|}
\hline \multicolumn{6}{|c|}{ Parámetros } \\
\hline Zona & Categoría & Ecuación & CR & b & $\mathbf{R}^{2}(\%)$ \\
\hline \multirow{2}{*}{ Providencia } & $\mathrm{H}$ & $0,844942+0,968163 * \mathrm{LC}$ & 0,84 & 0,96 & 71,5 \\
\hline & M & $0,406144+1,01827 * \mathrm{LC}$ & 0,94 & 1,01 & 89,3 \\
\hline \multirow{2}{*}{ I. Rosario } & $\mathrm{H}$ & $-0,789045+1,09839 * \mathrm{LC}$ & 0,96 & 1,09 & 93,2 \\
\hline & M & $-1,19321+1,14949 * \mathrm{LC}$ & 0,97 & 1,14 & 95,4 \\
\hline \multirow{2}{*}{ I. Tintipán } & $\mathrm{H}$ & $0,507965+1,06536 * \mathrm{LC}$ & 0,96 & 1,06 & 93,5 \\
\hline & M & $0,27421+1,02277 * \mathrm{LC}$ & 0,92 & 1,02 & 85,1 \\
\hline
\end{tabular}

Tabla 6. Análisis de regresión para las variables ancho del caparazón (AC) y peso (P) en Damithrax spinosissimus, del total por localidades $(\mathrm{H} / \mathrm{M})$ y por sexos $(\mathrm{M})$ Machos $(\mathrm{H})$ Hembras. Se da el coeficiente de correlación (CR), el valor de la pendiente (b) y el coeficiente de determinación $\left(\mathrm{R}^{2} \%\right)$.

\begin{tabular}{|c|c|c|c|c|c|}
\hline Zona & Categoría & Ecuación & $\mathbf{C R}$ & b & $\mathbf{R}^{2}(\%)$ \\
\hline \multirow[t]{3}{*}{ Providencia } & $\mathrm{H} / \mathrm{M}$ & $\ln \mathrm{P}=-0,531709+2,91839 \ln \mathrm{AC}$ & 0,76 & 2,91 & 58,7 \\
\hline & $\mathrm{H}$ & $\ln \mathrm{P}=0,976783+2,32642 \ln \mathrm{AC}$ & 0,58 & 2,32 & 33,6 \\
\hline & M & $\ln \mathrm{P}=-0,396915+2,90008 \ln \mathrm{AC}$ & 0,83 & 2,90 & 69,8 \\
\hline \multirow[t]{3}{*}{ I. Rosario } & $\mathrm{H} / \mathrm{M}$ & $\ln \mathrm{P}=-0,0665353+2,63793 \ln \mathrm{AC}$ & 0,92 & 2,63 & 84,9 \\
\hline & $\mathrm{H}$ & $\ln \mathrm{P}=0,411411+2,41005 \ln \mathrm{AC}$ & 0,91 & 2,41 & 83,2 \\
\hline & M & $\ln \mathrm{P}=-0,0847129+2,65846 \ln \mathrm{AC}$ & 0,91 & 2,65 & 83,9 \\
\hline \multirow[t]{3}{*}{ I. Tintipán } & $\mathrm{H} / \mathrm{M}$ & $\ln \mathrm{P}=-1,06221+3,07535 \ln \mathrm{AC}$ & 0,94 & 3,10 & 89,5 \\
\hline & $\mathrm{H}$ & $\ln \mathrm{P}=0,436762+3,12075 \ln \mathrm{AC}$ & 0,91 & 3,10 & 83,1 \\
\hline & M & $\ln \mathrm{P}=-0,785522+2,9728 \ln \mathrm{AC}$ & 0,94 & 2,97 & 88,6 \\
\hline
\end{tabular}


Con respecto a las variables de $\mathrm{AC}$ y $\mathrm{P}$, los machos presentaron un promedio mayor que las hembras, lo que coincide con lo señalado por Tunberg \& Creswell (1991) y Guzmán \& Tewfik (2004) en D. spinosissimus y otras especies de Brachyura (Carmona-Suárez 2003; Castillo, et al., 2011). Estas observaciones corroboran la presencia de dimorfismo sexual (machos $>$ hembras) registrada para la especie por Creswell, et al. (1989), especialmente en la superfamilia Majoidea, en la que el dimorfismo sexual se considera una regla (Baeza, et al., 2012) debido a la reducción del crecimiento somático en las hembras, lo que se ha asociado al hecho de que estas concentran su almacenamiento energético en el desarrollo gonadal (Mantelatto, et al., 2003), mientras que los machos alcanzan un mayor tamaño para competir exitosamente en la copulación de más de una hembra (Henmi, 2000). De acuerdo con Hernández-Reyes, et al. (2001), las razones para la diferencia de tallas entre machos y hembras de numerosas especies de cangrejos se debe posiblemente a: (1) la necesidad de que el macho sea mayor para asirla durante la cópula; (2) a diferentes tasas de crecimiento entre los sexos, y (3) a que las hembras detienen su crecimiento después de la muda de pubertad.

La longitud del cangrejo rey fue mayor en la Isla de Providencia que en las Islas del Rosario y Tintipán, lo que posiblemente se relaciona con mecanismos intrínsecos (como la información genética), y aquellos extrínsecos que ocasionan cambios en la estructura de las tallas en la población y dependen de la heterogeneidad espacial y temporal de las condiciones ambientales (Huston \& Angelis, 1987). Es así como las variables abióticas y la disponibilidad y calidad nutricional del alimento (Winfree \& Weinstein, 1986) en cada zona son determinantes en el tamaño que alcanza $D$, spinosissimus, como sucede en otras especies de Brachyura (Rodríguez, 1980; Buchanan \& Stoner, 1988). Este concepto está respaldado por las diferencias geomorfológicas, climáticas y oceanográficas que existen entre la Isla de Providencia y las Islas del Rosario y Tintipán, las cuales han sido descritas en el Caribe colombiano por Cendales, et al. (2002) y Sánchez, et al. (2005). Igualmente, estas diferencias de tallas entre las zonas muestreadas pueden estar relacionadas con la sobreexplotación del recurso y el uso de artes de pesca destructivos, ya que en Islas del Rosario y Tintipán gran parte de la población pesca con fines comerciales y de subsistencia (Bermúdez, et al., 2002), lo que puede llevar a la desaparición o la reducción de especies de importancia comercial y ecológica por acción de los pescadores y manejadores del área (Navas-Camacho, et al., 2009). En Providencia, en cambio, la pesca de la langosta y el cangrejo rey del Caribe no se da (observación personal, comunidad de pescadores).

La determinación de la proporción de los sexos en las especies es de interés biológico, pues permite detectar fluctuaciones en las poblaciones, migraciones reproductivas o alimenticias y el uso de diferentes hábitats por organismos de diferentes sexos (Wenner, 1972). En algunos estudios sobre Majoidea se ha señalado que en ocasiones la proporción sexual se desvía de la proporción esperada (M:H, 1:1), lo que puede estar relacionado con las técnicas de muestreo, las diferencias en la migración entre sexos, las actividades de agregación sexual, y la tasa de crecimiento o mortalidad (Monteiro-Teixeira, et al., 2008, 2009); sin embargo, en el presente trabajo la proporción de sexos de la especie no se diferenció significativamente de la esperada, lo cual también ha sido observado en otras especies de Majoidea (LópezGreco, et al., 2000), lo que posiblemente se relaciona con el proceso reproductivo continuo que pueden llegar a presentar estos organismos, como lo mencionan Baeza, et al. (2012).

A nivel de las poblaciones, la correlación de LC-AC y de AC-P presentó una relación alométrica positiva en donde b $\geq 3$, lo cual se ajusta a lo observado para $D$. spinosissimus en otras zonas del Caribe (Baeza, et al., 2012) y en otros braquiuros (Gould, 1971).

Por último, hubo una diferencia clara entre las tallas de los ejemplares de $D$. spinosissimus provenientes de la Isla de Providencia, en donde fueron mayores que las de los ejemplares de Islas del Rosario y de la Isla Tintipán. Los ejemplares provenientes de la Isla de Providencia tenían un mayor número de huevos que los de las otras dos estaciones debido a que habían alcanzado tallas mayores.

\section{Agradecimientos}

Los autores expresamos nuestros agradecimientos a Colciencias, por la financiación del proyecto "Evaluación del estado de las poblaciones del cangrejo rey del Caribe, $M$. spinosissimus (Lamarck, 1818), un modelo para el estudio de invertebrados bentónicos amenazados en Colombia”, código 1361-521-28198, así como a las sedes Caribe y Medellín de la Universidad Nacional de Colombia y a la Universidad de Cartagena, por sus aportes. A la Unidad Administrativa Especial de Parques Nacionales Naturales McBeen Old Prividence y a su directora Marcela Cano, a la de Islas del Rosario y San Bernardo y al biólogo Esteban Zarsa, por su apoyo y por habernos permitido muestrear en esas áreas de reserva en el marco del convenio entre la UAEPNN y la Universidad Nacional de Colombia, e, igualmente, a los pescadores de Providencia e Islas de Rosario por su apoyo durante los muestreos. Contribución No. 421 del Instituto de Estudios en Ciencias del Mar CECIMAR de la sede Caribe de la Universidad Nacional de Colombia.

\section{Conflicto de Intereses}

Los autores manifestamos no presentar ningún conflicto de intereses frente a la información presentada en el presento documento. 


\section{Bibliografía}

Arana, P. 2000. Estimación de abundancia y biomasa del cangrejo dorado (Chaceon chilensis), en el archipiélago de Juan Fernández, Chile. Invest. Mar., Valparaíso. 28: 53-68.

Baeza, J. A., Andreson, J .R., Spadaro, A. J., Behringer, D. C. 2012. Sexual dimorphism, allometry, and size at first maturity of the Caribbean king crab, Mithrax spinosissimus, in the Florida Keys. J. Shellfish Res. 31 (4): 909-916.

Bermúdez, A., Campos N. H., Navas G. R. 2002. Crustáceos. En N. Ardila, G. R. Navas y J. Reyes (Editores). Libro rojo de los invertebrados marinos de Colombia. INVEMAR. Ministerio del Medio Ambiente. La serie Libros rojos de especies amenazadas de Colombia. Bogotá, Colombia. p. 101-122.

Buchanan, B. A. \&. Stoner W. A. 1988. Distributional patterns of blue crabs (Callinectes sp.) in a tropical estuarine lagoon. Estuaries. 11: 231-239.

Castillo, J., Eslava, N., González, L. W. 2011. Crecimiento del cangrejo Callinectes danae (Decapoda: portunidae) de la Isla de Margarita Venezuela. Rev. Biol. Trop. 59 (4): 1525-1535.

Carmona-Suárez, C. A. 2003. Reproductive biology and relative growth in the spider crab Maja crispata (Crustacea: Brachyura: Majidae). Sci. Mar. 67: 75-80.

Cendales, M. H., Sea, S., Díaz, J. M. 2002. Geomorfología y unidades ecológicas del complejo de arrecifes de las Islas del Rosario e Isla Barú (Mar Caribe, Colombia). Rev. Acad. Colomb. Cienc. 26 (101): 497-510

Creasey, S., Rogers, A. D., Tyler, P. A., Young C. M., Gage J. D. 1997. The population biology and genetics of the deep-sea spider crab Encephaloides armstrongi Wood-Mason 1891 (Decapoda: Majidae). Philos. Trans. R. Soc. London Ser. 352B: 365-379.

Creswell, R., Tunberg, B. G., Winfree, R. A. 1989. Mariculture of the Caribean king crab Mithrax spinosissimus (Lamarck), in the Caribbean region: Progress and constraints. Proceedings of the $39^{\text {th }}$ Gulf and Caribbean Fisheries Institute. 469- 476.

Cruz, N. \& Campos, N. H. 2000. Los cangrejos araña (Decapoda:Brachyura: Majoidea) del Caribe colombiano. Biota colombiana. 4: 261-269.

Gould, S. J. 1971. Geometric similarity in allometric growth: A contribution to the problem of scaling in the evolution of size. Am. Nat. 105: 113-136.

Guzmán, M. H. \& Tewfik, A. 2004. Population characteristics and co-occurrence of three exploited decapods (Panulirus argus, $P$. guttatus and Mithrax spinosissimus) in Bocas del Toro, Panamá. J. Shellfish Res. 23 (2): 575-580.

Henmi, Y. 2000. Comparisons of life history traits among populations of the Ocypodid crab Macrophthalmus japonicus in habitats with contrasting food availability. Crust. Res. 29: 109-120.

Hernández-Reyes I. M., Palazón-Fernández, J. L., BolañosCurvelo, J. A., Hernández J. E. 2001. Aspectos reproductivos de Mithrax forceps (A. Milne-Edwards, 1875) (Crustacea: Decapoda: Majidae). Ciencias Marinas. 27 (1): 21-34.
Hultgren, K. M. \& Stachowicz, J. J. 2008. Molecular phylogeny of the brachyuran crab superfamily Majoidea indicates close congruence with trees based on larval morphology. Mol. Phylogenet. Evol. 48: 986-996.

Huston, M. A. \& Angelis, D. L. 1987. Size bimodality in monospecific polutations: A critical review of potential mechanisms. Am. Nat. 129 (5): 678-707.

Huxley J.S. 1950. Relative growth and form transformation. Proceedings of the Royal Society of London (B). 137: 465469.

Jones, M. B. \& Simons, M. J. 1983. Latitudinal variation in reproductive characteristics of a mudcrab, Helice crassa (Grapsidae). Bull. Mar. Sci. 33: 656-670.

López-Greco, L. S., Hernández, J. E., Bolaños, J. E., Rodríguez, E. M., Hernández, G. 2000. Population features of Microphrys bicornutus Latreille, 1825 (Brachyura, Majidae) from Isla Margarita, Venezuela. Hydrobiologia. 439: 151-159.

Merchán-Cepeda, A., Campos, N. H., Franco, A., Bermúdez, A. 2009. Distribución y datos biológicos de los cangrejos ermitaños (Decapoda: Anomura) del mar Caribe colombiano colectados por la expedición Invemar-Macrofauna II. Bol. Invest. Mar. Cost. 38 (1): 121-142.

Mantelatto, F., Faria, F., García, R. 2003. Biological aspects of Mithraculus forceps (Brachyura: Mithracidae) from Anchieta Island, Ubatuba, Brazil. J. Mar. Biol. Ass. U.K. 83: 789-791.

Monteiro-Teixeira, G., Franzoso, V., Castilho, A. L., Da Costa, R. C., De Morais-Freire, F. A. 2008. Size distribution and sex ratio in the spider crab Epialtus brasiliensis (Dana 1852) associated with seaweed on a rocky shore in southeastern Brazil (Crustacea, Decapoda, Brachyura, Majoidea, Epialtidae). Senckenberg. Biol. 88 (2): 169-176.

Monteiro-Texeira, G, Franzoso, V., Cobo V. J., Hiyodo, C. M. 2009. Population features of the spider crab Acanthonyx scutiformis (Dana1851) (Crustacea, Majoidea, Epialtidae) associated with rocky-shorealgae from southeastern Brazil. PANAMJAS. 4 (1): 87-95.

Navas-Camacho, R., Gómez-Campo, K., Vega-Sequeda, J. C., López-Londoño, T. 2009. Estado de los arrecifes coralinos. 59-88. En INVEMAR (Editor). Informe del estado de los ambientes y recursos marinos y costeros en Colombia: año 2008. Serie de Publicaciones Periódicas No. 8, Instituto de Investigaciones Marinas y Costeras (INVEMAR), Santa Marta. p. 59-88

Pinheiro, M. A. A. \& Fiscarelli, A. G. 2009. Length-weight relationship and condition factor of the mangrove crab Ucides cordatus (Linnaeus, 1763) (Crustacea, Brachyura, Ucididae). Braz. Arch. Biol. Technol. 52: 397-406.

Provenzano, A. J. \& Brownell, W. 1977. Larval and early postlarval stages of the West Indian spider crab, Mithrax spinosissumus (Lamarck) (Decapoda: Majidae). Proc. Bio. Soc. Wash. 90 (3): 735-752.

Rodríguez, G. 1980. Los crustáceos decápodos de Venezuela. Instituto Venezolano de Investigaciones Científicas, Caracas. Venezuela. 
Rodríguez, B. \& Hendrickx, M. 1992. Camarones, langostas y cangrejos. En F. Cervigón, R. Cipriani, W. Fischer, L. Garibaldi, M. Hendrickx, A.J. Lemus, R. Márquez, J. M. Poutiers, G. Robaina y B. Rodríguez (Editores). Fichas FAO de identificación de especies para los fines de la pesca. Guía de campo de las especies comerciales marinas y de aguas salobres de la costa septentrional de Sur América. Preparado con el financiamiento de la comisión de Comunidades Europeas y de NORAD. Roma. p. 103-162.

Sánchez, J. A., Pizarro, V., Acosta, A. R., Castillo, P. A., Herrón, P., Martínez, J. C., Montoya P., Orozco C. 2005. Evaluating coral reef benthic communities in remote Caribbean atolls (Quitasueño, Serrana, and Roncador Banks) to recommend Marine-Protected Areas for the Sea Flower Biosphere Reserve. Atoll Res. Bull. 531: 1-65.

Sokal, R. R. \& Rohlf, F. J. 1995. Biometry: The principles and practice of statistics in biological research. New York, USA: W. H. Freeman.

Tewfik, A. \& Guzmán, H. M. 2003. Shallow-water distribution and population characteristics of Strombus gigas and Strombus costatus (Gastropod:Strombidae) in Bocas del Toro, Panamá. J. Shellfish. Res. 22: 789-794.
Tunberg, B. G. \& Creswell, R. L. 1991. Development, growth and survival in the juvenile Caribbean King crab Mithrax spinosissimus (Lamarck) reared in the laboratory. J. Crustac. Biol. 11: 138-149.

Vélez, M. M. 1978. Reporte sobre algunas especies de Majidae (Crustacea: Brachyura) para la costa Atlántica colombiana. An. Inst. Invest. Mar. Punta Betín. 9: 109-140.

Wenner, A. M. 1972. Sex ratio as a function of size marine crustacean. Am. Nat. 106: 321-350.

Williams, A. B. 1984. Shrimps lobsters, and crabs of the Atlantic coast of the United States, Maine to Florida. Washington, D.C., EE.UU: Smithsonian Institution Press.

Winfree, R. A. \& Weinstein, S. 1986. Food habitats of the Caribbean king crab Damithrax spinosissimus (Lamarck). Proceedings of the 39th Gulf and Caribbena Fisheries Institute. p. 458-464.

Windsor, A. M. \& Felder, D. L. 2014. Molecular phylogenetics and taxonomic reanalysis of the family Mithracidae MacLeay (Decapoda: Brachyura: Majoidea). Invertebrate Systematics. 28: 145-173. 